\title{
HURWITZ GROUPS: A BRIEF SURVEY
}

\author{
MARSTON CONDER
}

\begin{abstract}
Hurwitz groups are the nontrivial finite quotients of the $(2,3,7)$ triangle group $\left\langle x, y \mid x^{2}=y^{3}=(x y)^{7}=1\right\rangle$. This paper gives a brief survey of such groups, their significance, and some of their properties, together with a description of all examples known to the author.
\end{abstract}

\section{INTRODUCTION}

A Hurwitz group is any finite group which can be generated by an element $x$ of order 2 and an element $y$ of order 3 whose product $x y$ has order 7 . Equivalently, a Hurwitz group is any finite nontrivial quotient of the $(2,3,7)$ triangle group, that is, the infinite abstract group $\Delta$ with presentation $\Delta=\langle x, y| x^{2}=$ $\left.y^{3}=(x y)^{7}=1\right\rangle$. The significance of the latter group (and its quotients) is perhaps best explained by referring to some aspects of the theory of Fuchsian groups, hyperbolic geometry, Riemann surfaces, and triangle groups, as given below. Details may be found in the recent books by Beardon [1] and Jones and Singerman [15].

A Fuchsian group is any discrete subgroup of $\operatorname{PSL}(2, \mathbf{R})$, the group of all linear fractional transformations of the form $z \mapsto$ $(a z+b) /(c z+d)$ with $a, b, c, d \in \mathbf{R}$ satisfying $a d-b c=1$. The latter group acts on the upper half-plane $\mathscr{U}=\{z \in \mathbf{C} \mid \operatorname{Im}(z)>0\}$, in fact as the group of all conformal homeomorphisms of $\mathscr{U}$, and when $\mathscr{U}$ is endowed with the hyperbolic metric (given by $d s^{2}=\left(d x^{2}+d y^{2}\right) / y^{2}$ for $\left.z=x+i y \in \mathbf{C}\right), \mathscr{U}$ becomes a model of the hyperbolic plane, and $\operatorname{PSL}(2, \mathbf{R})$ acts as a group of hyperbolic isometries.

Any given Fuchsian group $\Gamma$ acts properly discontinuously on $\mathscr{U}$, and the quotient space $S=\mathscr{U} / \Gamma$ is a Riemann surface. Conversely, every Riemann surface can be obtained in this way. A fundamental region for $\Gamma$ is a closed set $F$ in $\mathscr{U}$ whose $\Gamma$-images

Received by the editors July 31,1988 .

1980 Mathematics Subject Classification (1985 Revision). Primary 20F05; Secondary $57 \mathrm{~S} 25$. 
$\{\gamma(F) \mid \gamma \in \Gamma\}$ cover $\mathscr{U}$ and have mutually disjoint interiors; for example, if $\rho$ is the hyperbolic metric, and $w$ is any point of $\mathscr{U}$, then $F$ may be taken as $\{z \in \mathscr{U} \mid \rho(z, w) \leq \rho(z, \gamma(w))$ for all $\gamma \in \Gamma\}$, the Dirichlet region centered at $w$. The Riemann surface $S=\mathscr{U} / \Gamma$ is compact if and only if $\Gamma$ has a compact Dirichlet region. Also if $S$ is compact, then $\Gamma$ has no parabolic elements.

In any case, if $\Gamma$ has a Dirichlet region $F$ with finite hyperbolic area, then $\Gamma$ is a finitely generated group, with a presentation in terms of $r$ elliptic generators $x_{1}, x_{2}, \ldots, x_{r}$, and $s$ parabolic generators $p_{1}, p_{2}, \ldots, p_{s}$, and $2 t$ hyperbolic generators $a_{1}, b_{1}, a_{2}, b_{2}, \ldots, a_{t}, b_{t}$, subject only to the relations $x_{1}^{m_{1}}=x_{2}^{m_{2}}=\cdots=x_{r}^{m_{r}}=1$ along with the single additional relation $x_{1} x_{2} \ldots x_{r} p_{1} p_{2} \ldots p_{s} a_{1} b_{1} a_{1}^{-1} b_{1}^{-1} a_{2} b_{2} a_{2}^{-1} b_{2}^{-1} \ldots a_{t} b_{t} a_{t}^{-1} b_{t}^{-1}=$ 1. The hyperbolic area of $F$ is then given by $\mu(F)=$ $2 \pi\left\{(2 t-2)+\sum_{j=1}^{r}\left(1-1 / m_{j}\right)+s\right\}$.

Now if $S$ is any compact Riemann surface whose genus is 2 or more, then also $S=\mathscr{U} / K$ for some Fuchsian group $K$ having no elliptic elements (and no parabolic elements), indeed with presentation

$$
\begin{aligned}
\left\langle a_{1}, b_{1}, a_{2}, b_{2}, \ldots, a_{g}, b_{g}\right| a_{1} b_{1} a_{1}^{-1} b_{1}^{-1} & a_{2} b_{2} a_{2}^{-1} b_{2}^{-1}, \\
& \left.\ldots a_{g} b_{g} a_{g}^{-1} b_{g}^{-1}=1\right\rangle,
\end{aligned}
$$

and the hyperbolic area of a Dirichlet region for $K$ is $2 \pi(2 g-2)$. The group Aut $S$ of all automorphisms (that is, conformal homeomorphisms) of $S$ is then isomorphic to $\Gamma / K$ for some Fuchsian group $\Gamma$ containing $K$ as a normal subgroup; and because the index of $K$ in $G$ is $2 \pi(2 g-2) / \mu(F)$, where $\mu(F)$ is the hyperbolic area of some compact Dirichlet region $F$ for $\Gamma$ in $\mathscr{U}$, it follows that Aut $S$ is finite.

In particular, from the formula given above (with $s=0$ ), the smallest positive value for $\mu(F)$ is $\pi / 21$, obtainable only when $t=0$ and $r=3$ and $\left\{m_{1}, m_{2}, m_{3}\right\}=\{2,3,7\}$. This leads to the famous theorem of Hurwitz [14], namely, that if $S$ is any compact Riemann surface of genus $g \geq 2$, then $\mid$ Aut $S \mid \leq$ $84(g-1)$. The bound is attained precisely when $\Gamma$ has the presentation $\left\langle x_{1}, x_{2}, x_{3} \mid x_{1}^{2}=x_{2}^{3}=x_{3}^{7}=x_{1} x_{2} x_{3}=1\right\rangle$, that is, when $\Gamma$ is isomorphic to the $(2,3,7)$ triangle group $\Delta=\langle x, y| x^{2}=$ $\left.y^{3}=(x y)^{7}=1\right\rangle$, in which case Aut $S$ is a Hurwitz group! Obviously Hurwitz groups derive their name from this result. 
More generally, for positive integers $p, q$ and $r$, the $(p, q, r)$ triangle group is the group with presentation $\langle x, y| x^{p}=y^{q}=$ $\left.(x y)^{r}=1\right\rangle$. When $1 / p+1 / q+1 / r>1$, this group is finite, and is one of the finite spherical groups (either cyclic or dihedral, or isomorphic to $A_{4}, S_{4}$, or $A_{5}$ ); in particular the 2-sphere can be tessellated using a triangle whose interior angles are $\pi / p, \pi / q$, and $\pi / r$. When $1 / p+1 / q+1 / r=1$, the group is infinite but soluble (in fact Abelian-by-cyclic, c.f. [10]), and the euclidean plane can be tessellated using a triangle with angles $\pi / p, \pi / q$, and $\pi / r$. Finally, if $1 / p+1 / q+1 / r<1$, then the $(p, q, r)$ triangle group is infinite but insoluble, and the hyperbolic plane can be tessellated using a hyperbolic triangle with angles $\pi / p, \pi / q$, and $\pi / r$.

The importance of the $(2,3,7)$ triangle group $\Delta$ comes from the fact that the smallest nonnegative value of the expression $1-$ $(1 / p+1 / q+1 / r)$ is attained when $\{p, q, r\}=\{2,3,7\}$.

In particular, an insoluble group $G$ (other than $A_{5}$ ) cannot be a quotient of any of the spherical or euclidean triangle groups, and so the best possible generating pair $\{x, y\}$ for any such group (in terms of minimality of the orders of generators and their product) would be one satisfying the relations $x^{2}=x^{3}=(x y)^{7}=1$, if such a pair exists.

For the same reasons, quotients of the $(2,3,7)$ triangle group have importance also in the study of regular maps. A regular map of type $\{p, q\}$ on a surface of Euler characteristic $\chi$ is essentially a map (that is, a graph embedded in the surface with faces homeomorphic to the unit disk) whose automorphism group $G$ acts regularly on the set of ordered edges, so that every face of the map is surrounded by $p$ edges, and every vertex is incident with $q$ edges. If such a map has $n$ vertices, then it has $n q / 2$ edges and $n q / p$ faces, and therefore $\chi=n-n q / 2+n q / p=|G|(1 / q-1 / 2+1 / p)$. Hence the maps with the largest possible number of automorphisms on a given surface occur only when the quantity $1 / q+1 / p$ takes its largest value less than $1 / 2$, namely $10 / 21$; that is, when $\{p, q\}=\{3,7\}$. Correspondingly $G$ can be generated by an element of order 2 (reflecting any chosen edge), and one of order 3 (permuting the edges around either a face or a vertex), such that their product has order 7 ; in other words, $G$ is a Hurwitz group!

In this paper a survey is given of much that is known about these groups. Section 2 begins with some elementary properties of Hurwitz groups in general, and includes a few devices that are 
useful in the search for them. Section 3 gives a list of all simple Hurwitz groups known to the author, and Section 4 concludes the paper by describing some larger examples, together with methods for constructing such things.

\section{HURWITZ GROUPS: ELEMENTARY PROPERTIES}

Suppose $G$ is any Hurwitz group. The following properties are immediate:

(a) $G$ is perfect (and therefore insoluble),

(b) $G$ has a maximal normal subgroup $K$ such that $G / K$ is a non-Abelian simple Hurwitz group, and

(c) the order of $G$ is divisible by 84 .

The first is a consequence of the fact that the Abelianization of the $(2,3,7)$ triangle group $\Delta$ is trivial-if $x$ and $y$ commute, then the relations $x^{2}=y^{3}=(x y)^{7}=1$ collapse to $x=y=$ 1. In particular, $G$ cannot be soluble, for it has no composition series with Abelian factors. Next, if $K$ is any maximal normal subgroup of $G$, then $G / K$ is simple and, being also a nontrivial quotient of $\Delta$, must also be a Hurwitz group. The last property is a consequence of Hurwitz's theorem, although it can also be proved more directly (as in [21] for example).

These properties make it clear that a sensible way to begin any search for Hurwitz groups is to look at finite simple groups (and, in particular, those whose orders are divisible by 84). Such an approach was adopted by the author of this paper, in finding all Hurwitz groups of order less than a million (see [7]).

The smallest Hurwitz group is the simple group $\operatorname{PSL}(2,7)$, of order 168, which is the group of Klein's quartic curve $x^{3} y+y^{3} z+$ $z^{3} x=0$. It can be generated (for instance) by the linear fractional transformations $z \mapsto-1 / z$ and $z \mapsto(z-1) / z$ of the projective line over the field $\mathbf{Z}_{7}$, these transformations having orders 2 and 3 , respectively, with their product being $z \mapsto z+1$ (which has order 7). In fact, $\operatorname{PSL}(2,7)$ is known to have the presentation $\left\langle x, y \mid x^{2}=y^{3}=(x y)^{7}=[x, y]^{4}=1\right\rangle$, where $[x, y]$ denotes the commutator of $x$ and $y$. Presentations similar to this one, that is, with an extra relator inserted, have been considered by several authors (see [9, 17, 19] and [29]). In particular, from their work we can extract the following:

(d) If $\{x, y\}$ is a generating pair for a group $G$ satisfying the relations $x^{2}=y^{3}=(x y)^{7}=1$, and $m$ is the or- 
der of the commutator $[x, y]$, then $G$ is trivial if $m=$ $1,2,3$, or 5 , or isomorphic to $\operatorname{PSL}(2,7)$ if $m=4$, or to $\operatorname{PSL}(2,13)$ if $m=6$ or 7 , or to a nonsplit extension by $\operatorname{PSL}(2,7)$ of an elementary Abelian group of order 64 if $m=8$.

Also, if $m=9$, then the group $G$ must be an extension by $\operatorname{PSL}(2,8)$ of an Abelian or center-by-Abelian group, according to calculations by Leech in [19] based on the discovery by Sims that the group with presentation $\left\langle x, y \mid x^{2}=y^{3}=(x y)^{7}=[x, y]^{9}=1\right\rangle$ is infinite.

The situation is not fully known for larger values of $m$, but it seems likely that the corresponding presentations define infinite groups in those cases too, and of course in many cases a number of finite factor groups have been found. For example, the simple groups $\mathrm{J}_{1}$ and $\operatorname{PSL}(2,113)$ both have generating pairs $\{x, y\}$ satisfying $x^{2}=y^{3}=(x y)^{7}=[x, y]^{19}=1$ (see [13]).

Turning now from group presentations to permutation representations, we have this very useful property:

(e) If $\{x, y\}$ is a generating pair for a group $G$ satisfying the relations $x^{2}=y^{3}=(x y)^{7}=1$, then in any transitive permutation representation of $G$ on a set of size $N$, if the permutation induced by $x$ is made up of $k$ transpositions (and $n-2 k$ fixed points), and the permutations induced by $y$ and $x y$ have a total of $\gamma$ and $\eta$ cycles (including 1 -cycles, that is, fixed points, as well as 3-or 7-cycles), respectively, then $k \geq \gamma+\eta-2$. In particular, a necessary condition on $N$ is that $[N / 2]+2[N / 3]+6[N / 7] \geq 2 N-2$.

For a proof of the same sort of thing in a more general setting, see [8] and other references listed there. For an application, consider the Mathieu group $\mathbf{M}_{23}$ : in the natural action of this group on 23 points, every involution has cycle type $1^{7} 2^{8}$, every element of order 3 has cycle type $1^{5} 3^{6}$, and every element of order 7 has cycle type $1^{2} 7^{3}$, but if $k=8$ and $\gamma=11$ and $\eta=5$, then $k<\gamma+\eta-2$; therefore $\mathrm{M}_{23}$ is not a Hurwitz group. The same argument can also be applied to the sporadic simple groups HS (Higman-Sims) and McL (McLaughlin) in their representations on 100 and 275 points, respectively: neither of them is a Hurwitz group.

Another useful device for detecting pairs of generators of the sort in question is provided by a well-known result from character 
theory (c.f. Theorem 2.12 in [12]):

(f) If $z$ is an element of order 7 in the finite group $G$, and $K$ and $L$ are conjugacy classes of elements of $G$ such that $K$ contains involutions and $L$ contains elements of order 3 , then the number of ways in which $z$ can be expressed as a product $x y$ with $x \in K$ and $y \in L$ is given by

$$
\frac{|K||L|}{|G|} \sum_{i=1}^{m} \frac{\chi_{i}(K) \chi_{i}(L) \overline{\chi_{i}(z)}}{\chi_{i}(1)},
$$

where $\chi_{1}, \chi_{2}, \ldots, \chi_{m}$ are the ordinary (complex) characters of $G$, and $\chi_{i}(K)$ and $\chi_{i}(L)$ denote the values of the character $\chi_{i}$ on the classes $K$ and $L$, and $\chi_{i}(1)$ its degree.

For example, in the group $\operatorname{PSU}(3,3)$ this formula shows there are precisely seven pairs $\{x, y\}$ with $x$ of order 2 , and $y$ of order 3 , and $x y$ equal to a given element of order 7 . But also the same thing is true in the group $\operatorname{PSL}(2,7)$, and as this is isomorphic to a subgroup of $\operatorname{PSU}(3,3)$, and all cyclic subgroups of order 7 are conjugate in the latter group, it follows that every such pair generates a subgroup isomorphic to $\operatorname{PSL}(2,7)$. In particular, $\operatorname{PSU}(3,3)$ is not a Hurwitz group, contrary to a suggestion made in [27].

On the other hand, the same device was used in [13] and independently in [27] to show that the simple group $J_{1}$ is a Hurwitz group, and again in [27] to show that certain of the Ree groups are Hurwitz groups. Also it has often been fundamental to the enumeration of Hurwitz subgroups (among other things) in many of the larger sporadic finite simple groups. These will be discussed briefly in the next section.

\section{Simple Hurwitz Groups}

Relatively few of the non-Abelian simple groups are known to be quotients of the $(2,3,7)$ triangle group. Many small possibilities can be eliminated using the properties outlined in Section 2, but for the majority of the remainder a more sophisticated approach is likely to be required. Nevertheless three infinite families of simple groups have been found to be Hurwitz groups:

(1) The alternating group $A_{n}$ is a Hurwitz group, for all but finitely many positive integers $n$.

This was first proved by Graham Higman, using a clever argument based on the use of coset diagrams for the $(2,3,7)$ triangle 
group. The result remained unpublished until the author of this paper took it further, finding that in fact $A_{n}$ is a Hurwitz group for all $n \geq 168$, and for all but 64 integers $n$ in the range $3 \leq n \leq 167$ (see [4]). The exceptional values of $n$ are those which fail to satisfy the equality $[n / 2]+2[n / 3]+6[n / 7] \geq 2 n-2$ (c.f. Section 2 ), together with $7,8,9,14,16,24,30,44$, and 60 .

(2) The group $\operatorname{PSL}(2, q)$ is a Hurwitz group when $q=7$, and when $q=p$ for any prime $p \equiv \pm 1$ modulo 7 , and when $q=p^{3}$ for any prime $p \equiv \pm 2$ or \pm 3 modulo 7 , and for no other values of $q$.

This result is due to Macbeath (c.f. [24]), who showed in fact that $\operatorname{PSL}(2, q)$ has a Hurwitz subgroup whenever its order is divisible by 7 , but all such subgroups are mutually isomorphic.

(3) The simple Ree group ${ }^{2} G_{2}\left(3^{p}\right)$ is a Hurwitz group for every odd prime $p>3$.

The proof of this result (due to Chih-han Sah) may be found as part of Proposition 2.7 in [27]. Incidentally, Proposition 2.6 in [27] gives a classification of all simple Hurwitz groups having a Sylow 2-subgroup of order 8, based on theorems of Gorenstein and Walter.

Apart from these infinite families, eleven of the sporadic finite simple groups are known to be Hurwitz groups: the first Janko group $\mathbf{J}_{1}$ (see [13] or [27]); the Hall-Janko group $\mathbf{J}_{2}$ (see [11]); the smallest Conway group $\mathrm{Co}_{3}$ (see [32] or [30]); the Held group $\mathrm{He}$, the Rudvalis group $\mathrm{Ru}$, the Harada-Norton group $\mathrm{HN}$, and the Lyons group Ly (see [30] for all of these); the Fischer group $\mathrm{Fi}_{24}^{\prime}$ and the Thompson group Th (c.f. [22]); and the Fischer group $\mathrm{Fi}_{22}$ and the fourth Janko group $\mathrm{J}_{4}$ (c.f. [31]).

On the other hand, for instance, none of the Mathieu groups is a Hurwitz group: $M_{11}$ and $M_{12}$ have no elements of order 7; $\mathbf{M}_{22}$ and $\mathrm{M}_{23}$ can be eliminated using the condition cited as property (e) in Section 2; and $\mathbf{M}_{24}$ can be eliminated either by using a character-theoretic argument (just as for $\operatorname{PSU}(3,3)$ in Section 2, showing that every Hurwitz subgroup of $M_{24}$ has to be isomorphic to $\operatorname{PSL}(2,7))$, or, more simply, by verifying that the $(2,3,7)$ triangle group has only one transitive permutation representation of degree 24 , and that corresponds to the action of $\operatorname{PSL}(2,7)$ on cosets of a cyclic subgroup of order 7. (Note: there have been numerous mistaken claims that $\mathrm{M}_{24}$ is a Hurwitz group, usually resulting from a failure to identify $\operatorname{PSL}(2,7)$ as 
one of its maximal subgroups!) Of the remaining sporadic simple groups, all but the Baby Monster B and the Fischer-Griess Monster $\mathbf{M}$ (whose maximal subgroups are yet to be classified) have been shown to have no generating pairs satisfying the relations $x^{2}=$ $y^{3}=(x y)^{7}=1$ (see [31] and [16]).

Perhaps the only other fact worth mentioning here is this: the group $\operatorname{PSL}(3, q)$ is a Hurwitz group for no prime-power $q$ except 2 (in which case it is isomorphic to $\operatorname{PSL}(2,7)$ ) (c.f. [3]).

To conclude this section, here is a challenge question: is the Monster a Hurwitz group?

\section{CONSTRUCTIONS FOR LARGER HURWITZ GROUPS}

One of the easiest ways to construct large Hurwitz groups is to take a subdirect product of two smaller ones. In fact, if $K$ and $L$ are normal subgroups of finite index in the $(2,3,7)$ triangle group $\Delta$, and their intersection $M=K \cap L$ is properly contained in both, then the quotient $\Delta / M$ is a Hurwitz group isomorphic to a subgroup of the direct product $\Delta / K \times \Delta / L$ which projects onto each factor. Hence, for example, $\operatorname{PSL}(2,7) \times \operatorname{PSL}(2,8)$ is a Hurwitz group, as is $\operatorname{PSL}(2,13) \times \operatorname{PSL}(2,13)$, because there are two distinct normal subgroups of $\Delta$ giving $\operatorname{PSL}(2,13)$ as factor group; but, on the other hand, $\operatorname{PSL}(2,7) \times \operatorname{PSL}(2,7)$ is not, for there is only one normal subgroup of $\Delta$ giving $\operatorname{PSL}(2,7)$.

Another extremely fruitful way of finding composite Hurwitz groups was introduced by Macbeath in [23]. The idea here may be explained as follows:

If $K$ is a normal subgroup of finite index in the $(2,3,7)$ triangle group $\Delta$, with quotient $\Delta / K$ isomorphic to the Hurwitz group $G$ of order $84(g-1)$, then $G$ acts on a compact Riemann surface $S$ of genus $g$, whose fundamental group is isomorphic to $K$ (see Section 1). In particular, $K$ may be generated by $2 g$ distinct elements $a_{1}, b_{1}, a_{2}, b_{2}, \ldots, a_{g}, b_{g}$, these satisfying a single relation, namely, $\left[a_{1}, b_{1}\right]\left[a_{2}, b_{2}\right] \ldots\left[a_{g}, b_{g}\right]=1$. Hence, if $K^{\prime}$ denotes the commutator subgroup of $K$, then the quotient $K / K^{\prime}$ is a free Abelian group of rank $2 g$. But now for any positive integer $m$, if $K^{m}$ is the subgroup generated by the $m$ th powers of all the $a_{i}$ and $b_{i}$ (for $1 \leq i \leq g$ ), then the product $K^{\prime} K^{m}$ is a characteristic subgroup of $K$, such that the quotient $K / K^{\prime} K^{m}$ is an Abelian group of order $m^{2 g}$ (being a direct product of $2 g$ copies of the cyclic group of order $m$ ). And moreover, the group 
$\Delta / K^{\prime} K^{m}$ is an extension of this group by $G$, and therefore a Hurwitz group of order $84(g-1) m^{2 g}$, acting maximally on a compact Riemann surface of genus $(g-1) m^{2 g}+1$. In some cases, even a factor group of order $84(g-1) \mathrm{m}^{g}$ can be obtained, with a maximal action on a compact Riemann surface of genus $(g-1) m^{g}+1$ (c.f. [28]). See also [20].

This sort of approach was also taken by Leech in [18], giving presentations for several normal subgroups of $\Delta$ together with information about how the generators of $\Delta$ act by conjugation on these subgroups, and new presentations for some small Hurwitz groups.

Some years later Jeffrey Cohen used this information in order to completely classify all Hurwitz groups that are extensions by $\operatorname{PSL}(2,7)$ of an Abelian group: in each case the normal Abelian subgroup has to be a direct product of homocyclic groups of rank 3 or 6, depending on the exponent (see [2]). Furthermore, the same information can be used to construct a number of extensions by $\operatorname{PSL}(2,7)$ of a metabelian group, by analyzing the commutator subgroup of the normal subgroup $K$ for which $\Delta / K \cong \operatorname{PSL}(2,7)$, as in Section 4 of [7].

Also in [18] Leech raised the question as to whether a Hurwitz group could have a nontrivial center. He answered this question (affirmatively) himself in [19], by analyzing the normal subgroup of $\Delta$ corresponding to the case of $\operatorname{PSL}(2,8)$. In fact he showed that for every positive integer $p$, there is a Hurwitz group of order $504 p^{7}$ which is an extension by $\operatorname{PSL}(2,8)$ of an Abelian group of rank 7 and exponent $p$, and if $p$ is even, then the center of this group has order 2. Furthermore, if $p$ is divisible by 4 , there is another one of order $1008 p^{7}$ (being an extension of the cyclic group of order 2 by the first one), and this has center of order 4. A similar approach shows that for every positive integer $m$ divisible by 3 , there is a Hurwitz group of order $504 \mathrm{~m}^{7}$ which is an extension by $\operatorname{PSL}(2,7)$ of a metabelian group of order $3 \mathrm{~m}^{7}$, with a center of order 3 in each case (c.f. [5]).

Subsequently, the author of this paper discovered that the center of a Hurwitz group can be an arbitrarily large 2-group. The following construction shows how:

Take any ascending sequence of distinct odd prime-powers $\left\{q_{i}\right\}_{0 \leq i \leq s}$ satisfying the conditions given by Macbeath's result described in Section 3, and for $0 \leq i \leq s$, let $X_{i}$ and $Y_{i}$ be matri- 
ces which generate $\operatorname{SL}\left(2, q_{i}\right)$ and satisfy the relations $X_{i}^{2}=Y_{i}^{3}=$ $\left(X_{i} Y_{i}\right)^{7}=-I_{2}$ (where $I_{2}$ is the identity matrix). Let $F$ be the direct product of all these groups $\operatorname{SL}\left(2, q_{i}\right)$, define $X=X_{0} X_{1} \ldots X_{s}$ and $Y=Y_{0} Y_{1} \ldots X_{s}$, and let $R$ be the cyclic subgroup generated by $X^{2}$ (which is central in $F$ ). The factor group $F / R$ is then a Hurwitz group, generated by the cosets containing $X$ and $Y$, and its center is an elementary Abelian 2-group of order $2^{s}$.

The details may be found in [6].

Finally we mention that some interesting quotients of $\Delta$ may be found using a computer implementation of Sim's technique for finding all subgroups of prescribed low index in a given finitely presented group, using the CAYLEY system, for example. Enumerating subgroups of index up to 35 in $\Delta$ and examining the groups generated by the permutations induced by $x$ and $y$ on the cosets, one finds the following Hurwitz groups (as well as many other small examples already described): an extension of an elementary Abelian group of order $3^{7}$ by the Hurwitz group of order 1344 (itself an extension by $\operatorname{PSL}(2,7)$ of an elementary Abelian group of order 8), an extension by this group of an elementary Abelian group of order $2^{14}$, an extension by the alternating group $A_{15}$ of an elementary Abelian group of order $2^{14}$, and an extension by $\operatorname{PSL}(2,7)$ of the direct product of 7 copies of the alternating group $A_{5}$. The complete list (obtained from subgroups of index at most 40) is given in [25]; conjugacy classes of subgroups of index up to 50 were computed by Cannon as early as the mid-1970s (c.f. [26]).

\section{REFERENCES}

1. A. F. Beardon, The geometry of discrete groups, Springer-Verlag, Berlin, 1980.

2. J. M. Cohen, On Hurwitz extensions by $\operatorname{PSL}_{2}(7)$, Math. Proc. Cambridge Philos. Soc. 86 (1979), 395-400.

3. __ On non-Hurwitz groups and non-congruence subgroups of the modular group, Glasgow Math. J. 22 (1981), 1-7.

4. M. D. E. Conder, Generators for alternating and symmetric groups, J. London Math. Soc. (2) 22 (1980), 75-86.

5. __ A family of Hurwitz groups with non-trivial centres, Bull. Austral. Math. Soc. 33 (1986), 123-130.

6. _ Hurwitz groups with arbitrarily large centres, Bull. London Math. Soc. 18 (1986), 269-271.

7. __ The genus of compact Riemann surfaces with maximal automorphism group, J. Algebra 108 (1987), 204-247. 
8. M. D. E. Conder and J. McKay, A necessary condition for transitivity of a finite permutation group, Bull. London Math. Soc. 20 (1988), 235-238.

9. H. S. M. Coxeter, The abstract groups $G^{m, n, p}$, Trans. Amer. Math. Soc. 45 (1939), 73-150.

10. H. S. M. Coxeter and W. O. J. Moser, Generators and relations for discrete groups, 4th ed., Springer-Verlag, Berlin, 1980.

11. L. Finkelstein and A. Rudvalis, Maximal subgroups of the Hall-JankoWales group, J. Algebra 24 (1973), 486-493.

12. D. Gorenstein, Finite groups, 2nd ed., Chelsea Publishing Company, New York, 1980.

13. G. Higman, Construction of simple groups from character tables, Finite Simple Groups (M. B. Powell and G. Higman, eds.), Academic Press, New York, 1971, pp. 205-214.

14. A. Hurwitz, Über algebraische Gebilde mit eindeutigen Transformationen in sich, Math. Ann. 41 (1893), 403-442.

15. G. A. Jones and D. Singerman, Complex functions: an algebraic and geometric viewpoint, Cambridge Univ. Press, 1987.

16. P. B. Kleidman, R. A. Parker, and R. A. Wilson, The maximal subgroups of the Fischer group $\mathrm{Fi}_{23}$, J. London Math. Soc. (2) 39 (1989), 89-101.

17. J. Leech and J. Mennicke, Note on a conjecture of Coxeter, Proc. Glasgow Math. Assoc. 5 (1961), 25-29.

18. J. Leech, Generators for certain normal subgroups of $(2,3,7)$, Proc. Cambridge Philos. Soc. 61 (1965), 321-332.

19. __ Note on the abstract group $(2,3,7 ; 9)$, Proc. Cambridge Philos. Soc. 62 (1966), 7-10.

20. J. Lehner and M. Newman, On Riemann surfaces with maximal automorphism groups, Glasgow Math. J. 8 (1967), 102-112.

21. J. Lehner, Lectures on modular forms, Appl. Math. Ser., vol. 61, U. S. National Bureau of Standards, Washington, D.C., 1969.

22. S. A. Linton and R. A. Wilson, personal communication.

23. A. M. Macbeath, On a theorem of Hurwitz, Proc. Glasgow Math. Assoc. 5 (1961), 90-96.

24. __ Generators of the linear fractional groups, Number Theory, Proc. Sympos. Pure Math., vol. XII, Amer. Math. Soc., Providence, R.I., 1969, pp. 14-32.

25. J. Neubüser, H. Pahlings, and W. Plesken, CAS: Design and use of a system for the handling of characters of finite groups, Computational Group Theory (M. Atkinson, ed.), Academic Press, New York, 1984, pp. 195-247.

26. G. Rosenberger, Von Untergruppen der Triangel-Gruppen, Illinois J. Math. 22 (1978), 403-413.

27. C.-H. Sah, Groups related to compact Riemann surfaces, Acta Math. 123 (1969), 13-42.

28. D. Singerman, Automorphisms of compact non-orientable Riemann surfaces, Glasgow Math. J. 12 (1971), 50-59.

29. A. Sinkov, On the group-defining relations $(2,3,7 ; p)$, Ann. of Math. 38 (1937), 67-76.

30. A. J. Woldar, On Hurwitz generation and genus actions of sporadic groups, Illinois J. Math. 33 (1989), 416-437. 
31. __ Sporadic simple groups which are Hurwitz, preprint.

32. M. F. Worboys, Generators for the sporadic group $\mathrm{Co}_{3}$ as a $(2,3,7)$ group, Proc. Edinburgh Math. Soc. 25 (1982), 65-68.

Department of Mathematics and Statistics, University of Auckland, Private Bag, Auckland, New Zealand 